\title{
Electromagnetic Scattering Solution of Conducting Strips in Layered Media Using the Fast Multipole Method
}

\author{
Levent Gürel, Member, IEEE, and M. I. Aksun, Member, IEEE
}

\begin{abstract}
The fast multipole method (FMM) is applied to the solution of the electromagnetic scattering problems in layered media for the first time. This is achieved by using closedform expressions for the spatial-domain Green's functions for layered media. Until now, the FMM has been limited to the homogeneous-medium problems. An integral equation based on the two-dimensional scalar Helmholtz equation is solved to compute the electromagnetic scattering from sample geometries of conducting strips in layered media in order to demonstrate the accuracy and the efficiency of the new method.
\end{abstract}

\section{INTRODUCTION}

$\mathbf{N}$ UMERICAL solution of electromagnetic radiation and scattering problems involving layered media have gained popularity due to the need to computationally analyze and simulate various important geometries, e.g., microwave integrated circuits (MIC's), printed circuit boards (PCB's), and the vast class of microstrip-like structures. Numerical analysis and simulation of these structures are needed for both functional considerations and electromagnetic-compatibility (EMC) issues.

The formulation of layered-media problems has traditionally been carried out in the spectral domain due to the availability of the Green's functions in closed forms [1], [2]. Recently, a series of techniques have been developed to obtain closed-form Green's functions (CFGF's) for layered media in the spatial domain [3], [4]. The use of the CFGF's in a method-of-moments (MOM) formulation replaces the numerical computation of the improper integrals in the spectral domain with numerical integrations over finite regions in the spatial domain. Furthermore, the spatial domain integrals can be evaluated analytically in some cases [5]. Thus, this approach reduces the matrix-filling time by several orders of magnitude compared to the spectral-domain formulation. However, it does not reduce the computational complexities of the matrix-filling time and the memory requirement, which are both $O\left(N^{2}\right)$. Most importantly, despite the great savings in the matrix-filling time, the solution of the $N \times N$ dense matrix equation remains, which requires $O\left(N^{3}\right)$ operations

Manuscript received February 6, 1996. This work was supported in part by NATO's Scientific Affairs Division in the framework of the Science for Stability Programme and in part by the Scientific and Technical Research Council of Turkey (TUBITAK) under contracts EEEAG-132 and EEEAG163.

The authors are with the Department of Electrical and Electronics Engineering, Bilkent University, TR-06533, Bilkent, Ankara, Turkey

Publisher Item Identifier S 1051-8207(96)06021-7. in a direct scheme or $O\left(N^{2}\right)$ operations per iteration in an iterative scheme.

On another front, several researchers are working to reduce the computational complexities and the memory requirements of the solution of integral equations of electromagnetics. For the iterative solutions of the integral equations based on the Helmholtz equation, the fast multipole method (FMM), which has $O\left(N^{3 / 2}\right)$ complexity per iteration, has recently attracted attention [6], [7].

The FMM employs a harmonic expansion of the closedform Green's function and has so far been limited to the homogeneous-medium problems due to the lack of either the closed-form Green's function or the harmonic expansion thereof for other more complicated media, including the layered media. By obtaining a closed-form expression for the spatial domain Green's function for an arbitrarily layered medium and by interpreting each term of the expression as a discrete complex image, we have been able to apply the FMM to the solution of the Helmholtz equation for layered-media problems. Thus, we have obtained a fast solution techniques for the layered-media problems and extended the applicability of the FMM from homogeneous-medium problems to layered-medium problems. A similar technique has also been developed for the solution of Laplace equation [8].

\section{Fast Multipole Method in a LAyered Medium}

The FMM is a fast algorithm to compute the fields due to an arbitrary source distribution at a set of predetermined points in space. By formulating the solution of an integral equation in an iterative scheme, where one or more such field calculations are performed at each iteration, it becomes possible to reduce the complexity of the solution compared to traditional techniques.

The $y$-directed electric field at point $\rho=\hat{x} x+\hat{z} z$ due to a $y$-directed line source with unit amplitude located at point $\rho^{\prime}=\hat{x} x^{\prime}+\hat{z} z^{\prime}$ is given by

$$
\begin{aligned}
G\left(\rho, \rho^{\prime}\right)= & -\frac{\omega \mu}{4 \pi} \int_{-\infty}^{\infty} d k_{x} e^{i k_{x}\left(x-x^{\prime}\right)} \frac{1}{k_{z}} \\
& \cdot\left[e^{i k_{z}\left|z-z^{\prime}\right|}+\tilde{R}^{T E} e^{i k_{z}\left(z+z^{\prime}\right)}\right]
\end{aligned}
$$

where $\tilde{R}^{T E}$ is the generalized reflection coefficient defined at the $z=0$ plane due to an arbitrarily layered substrate below this plane. 


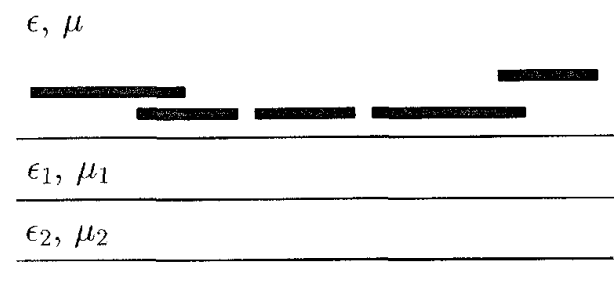

$\epsilon_{N}, \mu_{N}$

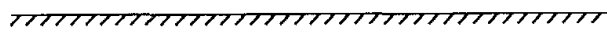
PEC

(a)

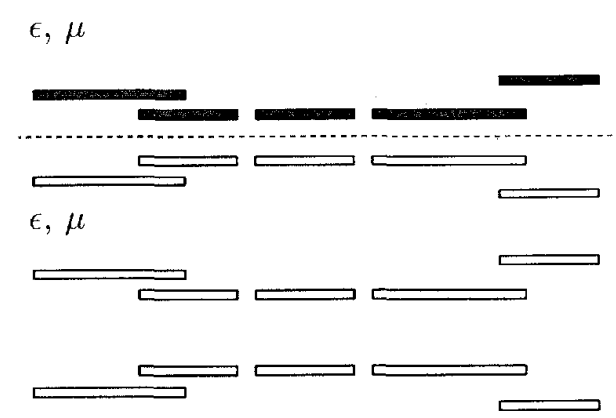

(b)

Fig. 1. (a) Original problem in a layered medium. (b) Equivalent problem with DCI's in a homogeneous medium.

Using a robust technique by Aksun [4], (1) can be converted to a closed-form expression given by

$$
\begin{aligned}
& G\left(\rho, \rho^{\prime}\right)= \\
& \quad-\frac{\omega \mu}{4}\left[H_{0}^{(1)}\left(k\left|\rho-\rho^{\prime}\right|\right)+\sum_{n=1}^{N_{I}} a_{n} H_{0}^{(1)}\left(k\left|\rho-\rho_{n}^{\prime}\right|\right)\right]
\end{aligned}
$$

where $\left|\rho-\rho_{n}^{\prime}\right|=\sqrt{\left(x-x^{\prime}\right)^{2}+\left(z+z^{\prime}+i \alpha_{n}\right)^{2}}$ and $a_{n}$ and $\alpha_{n}$ are complex constants for $n=1,2, \cdots, N_{I}$.

The FMM is based on the expansion of the Green's function using the addition theorems and no such expansions exist for the layered-media Green's functions given in (1) and (2). However, we can still employ the FMM in the solution of a layered-medium problem if we interpret (2) as the linear superposition of the field due to a source at $\rho^{\prime}$ and the fields due to $N_{I}$ discrete complex images (DCI's) located at $\rho_{n}^{\prime}=\hat{x} x^{\prime}+\hat{z}\left(-z^{\prime}-i \alpha_{n}\right)$. The DCI's are located at complex coordinates and, therefore, we need to use addition theorems for wave functions with complex arguments.

With the DCI interpretation, an equivalent problem is set up in a homogeneous medium. In this equivalent problem, which is illustrated in Fig. 1(b), $N_{I}$ image sources in a homogeneous medium are defined corresponding to each original source in the layered-medium problem [Fig. 1(a)]. Thus, if $N$ testing functions are defined on the original conductors, $N\left(N_{I}+1\right)$ basis functions are defined on the original conductors and their images.

The computation of the fields of $N\left(N_{I}+1\right)$ basis functions on the $N$ testing functions is carried out using the FMM

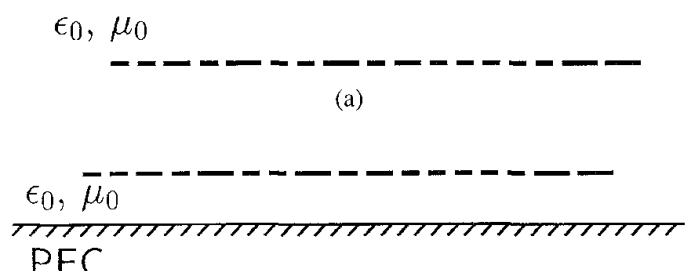

(b)

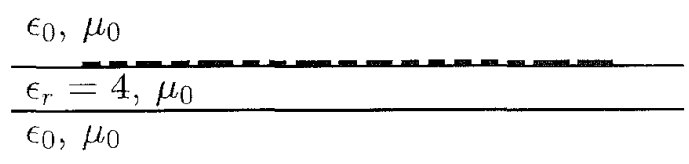

(c)

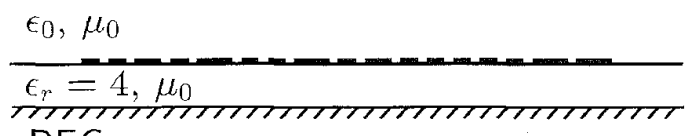

PEC

(d)

Fig. 2. Examples of layered structures.

and repeated several times in an iterative scheme. Since $N_{I}$ is a constant, this specific implementation of the FMM for layered-media problems has $O\left(N^{3 / 2}\right)$ computational complexity per iteration and $O\left(N^{3 / 2}\right)$ memory requirement as its homogeneous-medium counterpart.

\section{COMPutational Results}

In order to demonstrate the accuracy and the efficiency of the layered-media implementation of the FMM presented in this letter, a series of structures (as illustrated in Fig. 2), to which the method can be applied, have been designed. Common to all these structures is an irregular, finite, and planar array of strips, which has an overall extent of $1.5 \lambda_{0}$. Plane waves, whose electric fields are polarized in the $y$ direction and have unit amplitudes, are incident on the structures at $45^{\circ}$ as measured from the positive $x$ axis. Electromagnetic scattering problems for all four structures are solved using three different schemes: direct solution with Gaussian elimination, iterative solution with ordinary matrix-vector multiplication, and iterative solution with layered-medium implementation of the FMM. The numerical results obtained using these three different schemes agree with each other for several digits and, therefore, are indistinguishable on the plots presented in Fig. 3, thus testifying to the accuracy of the layered-medium implementation of the FMM.

The magnitude of the current distribution obtained on the array when the array is in free space [Fig. 2(a)] is shown in Fig. 3(a). An infinitely large conducting plane placed $\lambda_{0} / 100$ away from the array [Fig. 2(b)] causes the magnitude of the current distribution to increase as seen in Fig. 3(b). When the array is placed on a $\lambda_{0} / 100$ dielectric slab with $\epsilon_{r}=4$ [Fig. 2(c)], the magnitude of the current distribution, shown in Fig. 3(c), is seen to be modified and increased, but not as much as that of the conducting-plane case. If the dielectric 


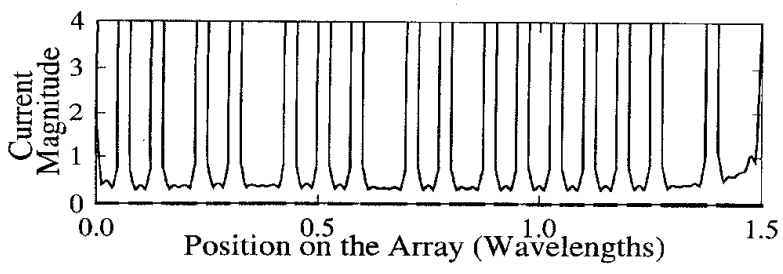

(a)

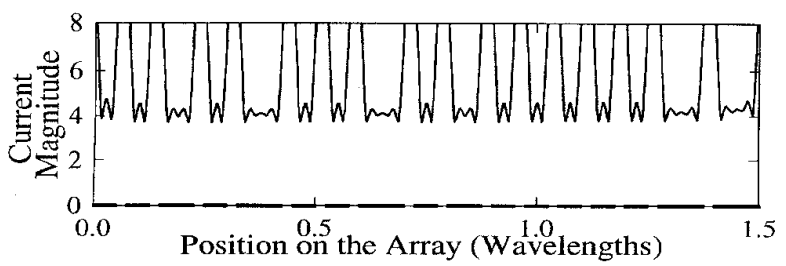

(b)

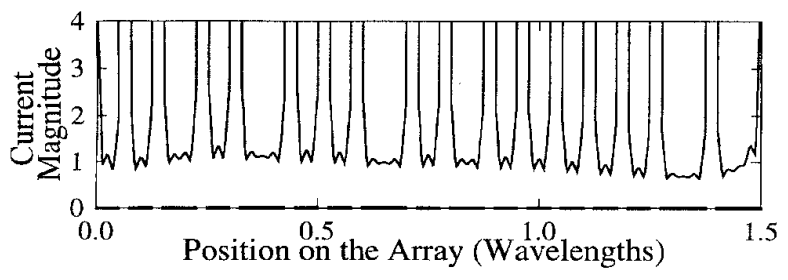

(c)

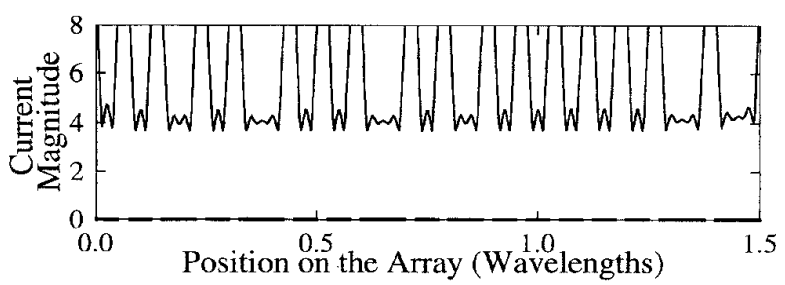

(d)

Fig. 3. Current distributions on the array corresponding to the example structures of Fig. 2.

slab is backed by a conducting plane as depicted in Fig. 2(d), the current magnitude becomes higher, as seen in Fig. 3(d). Indeed, Fig. 3(d) can be compared to Fig. 3(b) to conclude that the conducting plane is more dominant than the relatively thin dielectric slab in determining the current distribution. However, by comparing Fig. 3(a) and (c), it is easy to see that the dielectric slab has a significant effect on the current distribution in the absence of the conducting plane.

The discretization of the conducting array of Fig. 2(a) results in 105 basis and testing functions. This array is duplicated many times in the layered geometry of Fig. 2(d) to obtain problems that are ten times as large. Separating the solution and filling times, we have compared the solution times of the FMM and the traditional solution techniques. Fig. 4(a) compares the solution time of the FMM to the Gaussian elimination and Fig. 4(b) compares the CPU time required during a single iteration of the FMM to the ordinary matrixvector multiplication, respectively. As for the filling time, since

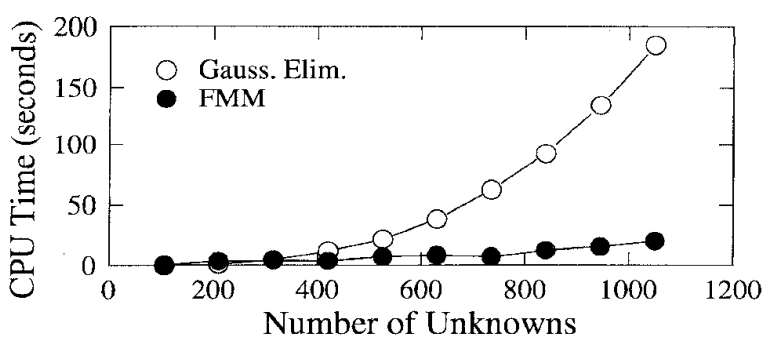

(a)

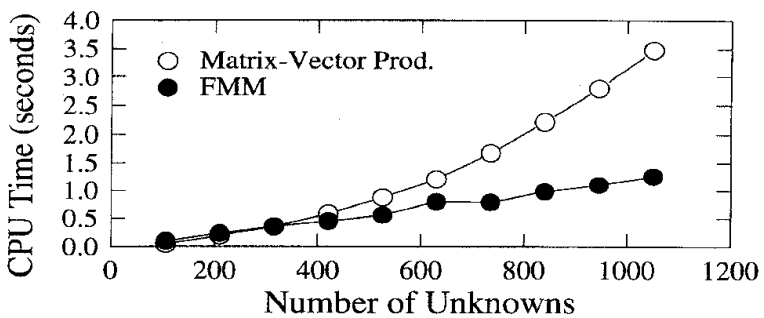

(b)

Fig. 4. (a) Comparison of the solution times of the layered-medium implementation of the FMM and the Gaussian elimination. (b) Comparison of the per-iteration solution times of the layered-medium implementation of the FMM and the ordinary matrix-vector multiplication.

only a sparse matrix of the near-field interactions is filled in the FMM as opposed to filling an $N \times N$ dense matrix in the direct solution, filling time of the FMM is always lower.

\section{CONCLUSION}

In this letter, the applicability of the FMM has been extended to layered-media problems. As an example, we have demonstrated the solution of the scalar Helmholtz equation for the electromagnetic scattering from a two-dimensional planar array of horizontal strips on a layered substrate. $\mathrm{Nu}$ merous straightforward generalizations are possible and will be reported elsewhere.

\section{REFERENCES}

[1] T. Itoh, "Spectral domain immittance approach for dispersion characteristics of generalized transmission lines," IEEE Trans. Microwave Theory Tech., vol. MTT-28, pp. 733-736, July 1980.

[2] W. C. Chew and L. Gürel, "Reflection and transmission operators for strips or disks embedded in homogeneous and layered media," IEEE Trans. Microwave Theory Tech., vol. MTT-36, pp. 1488-1497, Nov. 1988.

[3] Y. L. Chow, J. J. Yang, D. F. Fang, and G. E. Howard, "A closed-form spatial Green's function for the thick microstrip substrate," IEEE Trans. Microwave Theory Tech., vol. 39, pp. 588-592, Mar. 1991.

[4] M. I. Aksun, "A robust approach for the derivation of closed-form Green's functions," IEEE Trans. Microwave Theory Tech., vol. 44, pp. 651-658, May 1996.

[5] L. Alatan, M. I. Aksun, K. Mahadevan, and T. Birand, "Analytical evaluation of the MoM matrix elements," IEEE Trans. Microwave Theory Tech., to be published.

[6] V. Rokhlin, "Rapid solution of integral equations of scattering theory in two dimensions," J. Comput. Phys., vol. 86, pp. 414-439, Feb. 1990.

[7] C. C. Lu and W. C. Chew, "Fast algorithm for solving hybrid integral equations," Proc. IEE, vol, 140, pt. H, pp. 455-460, Dec. 1993.

[8] V. Jandhyala, E. Michiessen, and R. Mittra, "Multipole-accelerated capacitance computation for 3-D structures in a stratified dielectric medium using a closed-form Green's function," Int. J. Microwave Millimeter-Wave Computer-Aided Eng., vol. 5, pp. 67-87, Mar. 1995. 